Ophthalmologica 1975;171:494-504

\title{
Index rerum ad Vol. 171
}

\section{Confecit: C. Loeb-Schürch}

$(\mathrm{V})=$ Report - Vortrag - Communication $(\mathrm{B})=$ Book Reviews - Buchbesprechungen - Livres nouveaux

Abflussfazilität, v. Aqueous humour

dynamics Ablatio, v. Sponge implants Accidental coagulation; accidental laser coagulation of the central fovea $15(\mathrm{~V})$ Accommodation, v. Optic approach,

Phenylephrine ACTH, v. Metastatic carcinoma Acuité visuelle, v. Amblyopia, Optotype

chart, Photocoagulation, Visual acuity Adaptation à Гobscurité, v. Rod response Aderhaut, v.

Cysticercus, Metastatic

carcinoma, Retinal disturbances,

Trichomegaly Aging, v. Lipid composition, Yellowing Alterung, v. Lipid composition,

Yellowing Amblyopia; screening for amblyopia

in pre-school children 325 (V) Aniseikonia; unilateral aphakia and

tolerance of aniseikonia 258 (V) Anomalie de Peter, v. Peter's anomaly Anomalies; dystrophie

congénitale

héréditaire de la cornée associée à

des anomalies extraoculaires

diverses 410 Anterior chamber, v. Artificial lens,

Determination, Dioptric power,

Enucleation, Glaucoma-cataract

surgery, Mydriatiques, Stagnation

thrombosis, Ultrasonography

Antibiotics, v. Moraxella

Aphakia, v. Aniseikonia

Applanations-Tonometrie, v. Body position, Halothannarkose

Aqueous humour dynamics; investigations into the aqueous humour dynamics in primary pigmentary degeneration of the retina 165

Arcus lipoides, v. Lipid composition

Argon laser; low intensity argon laser coagulation in central serous retinopathy (CSR) 214

Artificial lens; special indications for the artificial lens $57(\mathrm{~V})$

Atrophie optique, v. Myambutol

Augeninnendruck, v. Aqueous humour dynamics, Body position, Cyclo-cryoapplication, Glaucoma-cataract surgery, Halothannarkose, Phenylephrine, Pump infusion

Automatic information processing in diabetic retinopathy 3 (V)

Bâtonnet, v. Rod response

Behcet's disease; treatment of Behcet's

disease with chlorambucil 347 (V) Bindehaut, v. Phagocytosis, Pterygium,

Sponge implants Binokularsehen, v. Aniseikonia, Depth

acuity, Kataraktchirurgie 
Index rerum ad Vol. 171

495

Biochemie, v. Glycoprotein, Lipid

composition, Phagocytosis, Vitreous

surgery Biometrie, v. Determination, Implant

lens, Ultrasonography Blocage pupillaire, v. Mydriatiques Body position; influence of body position

on the intraocular pressure of normal

and glaucomatous eyes 132 Book reviews 176 (B), 244 (B) Bronchuskarzinom, v. Metastatic

carcinoma Bruckner; Gedanken und Erinnerungen

an Arthur Bruckner 405 Buchbesprechungen 176 (B), 244 (B) Bullöse Keratitis, v. Enucleation

$\mathrm{C}$, v. Aqueous humour dynamics

Calcification cornéenne, v. Hyper-parathyroidism

Campimeter; preliminary results about a new remotely controlled projection campimeter 432

Canaliculus lacrimalis, v. Jones tube

Carcinoma, v. Metastatic carcinoma

Caruncle, v. Pterygium

Cataract, v. Artificial lens, Cataract extraction, Cataract surgery, Dog, First extractions,

Glaucoma-cataract surgery, Kataraktchirurgie, Lens research, Optic approach, Zonular cataract

Cataract extraction; panel discussion on cataract extraction 274 (V)

Cataract surgery in the course of the centuries 79 (V)

Central artery occlusion, v. Stagnation thrombosis

Central serous retinopathy, v. Argon laser

Central vein thrombosis, v. Photo-coagulation, Stagnation thrombosis

Chambre antérieure, v. Artificial lens, Determination, Dioptric power, Enucleation, Glaucomacataract surgery, Mydriatiques, Stagnation thrombosis, Ultrasonography

Champ visuel, v. Accidental coagulation, Campimeter, Photocoagulation, Static perimetry,

Visual acuity

Chiasma, v. Sparing of the macula

Chicken, v. Immunofluorescence

Chien, v. Dog

Chirurgie, v. Artificial lens, Cataract extraction, Cataract surgery, Corneal transplantation,

Cysticercus, Dog, Enucleation, Epikeratoprosthesis, First extractions, Fractures, Fuchs' corneal

dystrophy, Glaucoma-cataract surgery, Jones tube, Lid loading, Mucocele, Secondary glaucoma,

Sponge implants, Ultrasonography, Vitreous surgery

Chlorambucil, v. Behcet's disease

Chorioidea, v. Cysticercus, Metastatic carcinoma, Retinal disturbances, Trichomegaly

Chorioretinitis, v. Hodgkin's disease

Chromosom, v. Trichomegaly

Ciliary body, v. Cyclocryoapplication

Clinicopathologic case reports 236, 487

Closed angle glaucoma, v. Mydriatiques

Coagulation à la lumière, v. Accidental coagulation, Argon laser, Automatic information,

Diabetic retinopathy, Photocoagulation, Tunable laser

Cobaye, v. Phagocytosis 
Collagen, v. Vitreous surgery

Color blindness, v. Rod response

Color vision, v. Ethambutol

Complications oculaires, v. Enucleation, Ethambutol, Moraxella, Myambutol, Secondary

glaucoma

Computer, v. Automatic information, Campimeter, Implant lens

Cone, v. Rod response

Congrès, v. Netherlands Ophthalmological Society

Conjunctiva, v. Phagocytosis, Pterygium, Sponge implants

Conjunctivitis chronica, v. Phagocytosis

Contact lens, v. Aniseikonia, Epikeratoprosthesis

496

Index rerum ad Vol. 171

Cornea, v. Anomalies, Artificial lens, Corneal transplantation, Determination, Dioptric power, Epikerato-prosthesis, Fuchs' corneal dystrophy, Herpes keratitis, Herpetic keratitis,

Hyperparathyroidism, Keratocele, Lid loading, Lipid composition, Mydriatiques, Peter's

anomaly, Pterygium, Ultrasonography

Corneal dystrophy, v. Anomalies, Fuchs' corneal dystrophy

Corneal transplantation; perforating corneal transplantation: difficulties and possibilities 306 (V)

Corps ciliaire, v. Cyclocryoapplication

Corps vitré, v. Accidental coagulation, Cysticercus, Kataraktchirurgie, Vitreous surgery

Cortex, v. Sparing of the macula

Corticosteroids, v. Behcet's disease

Cristallin, v. Artificial lens, Enucleation, Epikeratoprosthesis, Immunofluores-cence, Implant

lens, Lens research, Mydriatiques, Optic approach, Peter's anomaly, Phenylephrine, Secondary

glaucoma, Surgical anatomy, Yellowing

Cryotherapy, v. Cyclocryoapplication

Cyclocryoapplication 332 (V)

Cyclofusion, v. Aniseikonia

Cysticercus; intraocular cysticercus 7(V)

Cystoid macular degeneration, v. Photocoagulation

Cytostatica, v. Behcet's disease

Daltonisme, v. Rod response Dark adaptation, v. Rod response Deafness, v. Anomalies

Décollement rétinien, v. Sponge

implants Dégénéreccence maculaire, v. Retinal

disturbances Dégénérescence tapétorétinienne,

v. Aqueous humour dynamics,

Trichomegaly

Depth acuity; comparability of two

methods of estimating real-depth

acuity 224 Descemet's membrane, v. Peter's

anomaly Determination of the expected power

of the implant lens by ultrasound

281 (V) Deuteranopie, v. Rod response Diabetes, v. Automatic information,

Diabetic retinopathy, Glycoprotein, 
Optic approach Diabetic retinopathy, v. Automatic information, Glycoprotein Diabetic retinopathy; treatment of diabetic retinopathy with photo-coagulation at advanced age 309 (V) Diagnose, v. Static perimetry Dialysis; retinal disturbances in renal dialysis patients $346(\mathrm{~V})$ Dioptric power of the lens implant 278 (V) Diplopieschwelle, v. Aniseikonia Dog; cataract extraction in the dog 296 (V) Donders lecture 1972, v. Vitreous surgery Donders medal; award of the Donders medal to Prof. J. Francois 386 (V) Drusen, v. Reticular structures Dunkeladaptation, v. Rod response Dystrophie cornéenne, v. Anomalies, Fuchs' corneal dystrophy Echographie, v. Determination, Implant lens, Ultrasonography Edema, v. Photocoagulation, Retinal disturbances EDTA, v. Hyperparathyroidism Elastosis, v. Reticular structures Electrooculogramm, v. Epitheliitis Electroretinogramm, v. Accidental coagulation, Rod response Elektronenmikroskopie, v. Metastatic carcinoma, Myxoma, Vitreous surgery Embryologie, v. Peter's anomaly Emmetropia, v. Implant lens

Index rerum ad Vol. 171

497

Emulsifikation, v. Kataraktchirurgie Endocrinology, v. Hyperparathyroidism, Metastatic carcinoma Endophthalmitis, v. Moraxella Enucleation; 'causes of enucleation' after lens extraction 267 (V) EOG, v. Epitheliitis Epikeratoprosthesis 46 (V) Epiphora, v. Jones tube Episcleral implants, v. Sponge implants Epitheliitis; acute retinal pigment epitheliitis 361 (V) Epithelinvasion, v. Enucleation Epithelium pigmentaire, v. Accidental coagulation, Argon laser, Epitheliitis,

Hodgkin's disease, Nyctalopia,

Reticular structures, Retinal disturbances, Tunable laser ERG, v. Accidental coagulation, Rod response Ethambutol; Farnsworth 100-hue test in diagnosis of ethambutol-induced damage of optic nerve 425 Exophthalmos 54 (V) Experimentelle Forschung, v. Cyclocryoapplication, Herpes keratitis, Phagocytosis, Tunable laser, Vitreous surgery Expulsivblutung, v. Enucleation Extraction du cristallin, v. Cataract extraction, Dog, Enucleation, First extractions, Glaucoma-cataract surgery, Secondary glaucoma Extravasate, v. Photocoagulation, Stagnation thrombosis

Facilité d'écoulement, v. Aqueous humour dynamics Farbblindheit, v. Rod response Farbsehen, v. Ethambutol Farnsworth 100-hue test,

v. Ethambutol First extractions; results of the first ten lens extractions 284 (V) Fixation disturbances; fundus television in the study of fixation disturbances 
$51(\mathrm{~V})$

Fluoroangiographie, v. Accidental coagulation, Argon laser, Behcet's disease, Dialysis, Epitheliitis, Metastatic carcinoma, Photocoagulation, Reticular structures, Stagnation thrombosis Fovea centralis, v. Accidental coagulation, Diabetic retinopathy, Epitheliitis, Photocoagulation, Sparing of the macula, Stagnation thrombosis

Fractures of the orbital roof $360(\mathrm{~V})$

Fredrickson; ocular anomalies in the types of Fredrickson $86(\mathrm{~V})$

Frequence sensitivity, v. Rod response

Fuchs' corneal dystrophy; keratoplasty in Fuchs' corneal dystrophy 96 (V)

Fundus, v. Automatic information, Cysticercus

Fundus television, v. Fixation disturbances

Fundus television; the technical background of fundus television $53(\mathrm{~V})$

Fusionsbreite, v. Aniseikonia

Gefässe, v. Behcet's disease, Diabetic retinopathy, Fredrickson, Photocoagulation, Retinal

disturbances, Stagnation thrombosis

Genetics, v. Anomalies, Aqueous humour dynamics, Fredrickson, Fuchs' corneal dystrophy,

Nyctalopia, Reticular structures, Retinoblastoma, Trichomegaly

Gesichtsfeld, v. Accidental coagulation, Campimeter, Photocoagulation, Static perimetry, Visual acuity

Glandes de Meibom, v. Pterygium

Glaskörper, v. Accidental coagulation, Cysticercus, Kataraktchirurgie, Vitreous surgery

Glaucoma, v. Aqueous humour dynamics, Artificial lens, Body position, Cyclocryoapplication, Mydriatiques, Peter's anomaly, Pump infusion, Secondary glaucoma, Stagnation thrombosis 498

Index rerum ad Vol. 171

Glaucoma-cataract surgery; combined glaucoma-cataract surgery 288 (V)

Glaucome à angle fermé,v. Mydriatiques

Glaucome à angle ouvert, v. Aqueous humour dynamics, Pump infusion

Glomerulonephritis, v. Dialysis

Glycoprotein; serum glycoprotein level as a possible early screening for the development of

diabetic retinopathy $316(\mathrm{~V})$

Gonioscopy, v. Aqueous humour dynamics, Mydriatiques

Greffe, v. Lid loading

Greffe cornéenne, v. Corneal transplantation, Fuchs' corneal dystrophy

Grönblad-Strandberg Syndrom, v. Reticular structures

Growth disturbances, v. Trichomegaly

Guinea pig, v. Phagocytosis

Halothannarkose; Augeninnendruck-messungen bei Kleinkindern ohne Glaukom in

Halothanmaskennarkose 202 Helminthiasis, v. Cysticercus Hemeralopia, v. Aqueous humour dynamics, Nyctalopia Hemorrhage, v. Accidental coagulation Hérédité, v. Anomalies, Aqueous humour dynamics, Fredrickson, Fuchs' corneal dystrophy, Nyctalopia, Reticular structures, Retinoblastoma, Trichomegaly Herpes keratitis; results of the treatment of experimental herpes keratitis with Poly I:C 69 (V) Herpes zoster, v. Hodgkin's disease Herpetic keratitis; the treatment of patients suffering from herpetic keratitis with Poly I:C and IDU 71 (V) 
Histochemie, v. Vitreous surgery Histologie, v. Hodgkin's disease, Metastatic carcinoma, Myxoma, Sarkom, Tunable laser, Vitreous surgery

Hodgkin's disease; ocular toxoplasmosis, trigeminal herpes zoster and pulmonary tuberculosis in a patient with Hodgkin's disease 237

Hormon, v. Metastatic carcinoma

Hornhaut, v. Anomalies, Artificial lens, Corneal transplantation, Determination, Dioptric power, Epikerato-prosthesis, Fuchs' corneal dystrophy, Herpes keratitis, Herpetic keratitis,

Hyperparathyroidism, Keratocele, Lid loading, Lipid composition, Mydriatiques, Peter's

anomaly, Pterygium, Ultrasonography

Huhn, v. Immunofluorescence

Humeur aqueuse, v. Aqueous humour dynamics

Hund, v. Dog

Hyalocytes, v. Vitreous surgery

Hyperlipoprotéinémie familiale, v. Fredrickson

Hyperparathyroidism; band keratopathy due to hyperparathyroidism 119

IDU, v. Herpes keratitis, Herpetic keratitis

Immunofluorescence; demonstration of lens proteins and serum proteins in the intraocular tissues of the chicken by means of immunofluorescence 266 (V) Immunology, v. Vitreous surgery

Immunosuppressiva, v. Behcet's disease Implantation du cristallin, v. Artificial lens,

Determination, Dioptric power, Implant lens, Kataraktchirurgie, Ultrasonography Implant lens;

the emmetropic and the iseikonic implant lens: computer calculation of the refractive power and its accuracy 467 Implants, v. Sponge implants Infection, v. Enucleation, Hodgkin's

disease, Moraxella Instrument, v. Body position, Pump

infusion, Tunable laser Intraocular pressure, v. Aqueous humour dynamics, Body position,

Cyclocryo-

Index rerum ad Vol. 171

499

application, Glaucoma-cataract surgery, Halothannarkose, Phenyl-ephrine, Pump infusion Invalidität, v. Visual acuity Invasion épithéliale, v. Enucleation Iris, v. Dog, First extractions, Glaucoma-cataract surgery, Mydriatiques, Peter's anomaly, Phenylephrine, Stagnation thrombosis Iris-clip-lens, v. Artificial lens Iseikonia, v. Dioptric power, Implant lens launissement, v. Yellowing

Jones tube; experiences with the Jones

tube in the Rotterdam eye clinic

$353(\mathrm{~V})$

Kammerwasser, v. Aqueous humour dynamics

Kaninchen, v. Cyclocryoapplication, Epikeratoprosthesis, Herpes keratitis, Tunable laser, Vitreous surgery

Karunkel, v. Pterygium

Katarakt, v. Artificial lens, Cataract extraction, Cataract surgery, Dog, First extractions, Glaucoma-cataract surgery, Kataraktchirurgie, Lens research, Optic approach, Zonular cataract

Kataraktchirurgie; über die Zukunft der Kataraktchirurgie 460

Keratitis bullosa, v. Enucleation

Keratitis herpetica, v. Herpes keratitis, Herpetic keratitis

Keratocele; ten years keratocele $95(\mathrm{~V})$ 
Keratopathy, v. Hyperparathyroidism

Keratoplastik, v. Corneal transplantation, Fuchs' corneal dystrophy

Kollagen, v. Vitreous surgery

Kongress, v. Netherlands Ophthalmological Society

Kontaktlinse, v. Aniseikonia, Epikeratoprosthesis

Körperlage, v. Body position

Kryotherapie, v. Cyclocryoapplication

Lacrimal pathways, v. Jones tube

Lageabhängigkeit, v. Body position

Lagophthalmus, v. Lid loading

Lapin, v. Cyclocryoapplication,

Epikeratoprosthesis, Herpes keratitis, Tunable laser, Vitreous surgery

Laser, v. Accidental coagulation, Argon laser, Tunable laser

Laurence-Moon-Bardet-Biedl Syndrome, v. Aqueous humour dynamics, Trichomegaly

Leakage, v. Photocoagulation, Stagnation thrombosis

Leaking point, v. Argon laser

Lehre, v. 'Student-assistancy'

Lens, v. Artificial lens, Enucleation, Epikeratoprosthesis, Immunofluores-cence, Implant lens, Lens research, Mydriatiques, Optic approach, Peter's anomaly, Phenylephrine, Secondary glaucoma, Surgical anatomy, Yellowing

Lens implantation, v. Artificial lens, Determination, Dioptric power, Implant lens,

Kataraktchirurgie, Ultrasonography

Lens research; advances in lens research 264 (V)

Lentille de contact, v. Aniseikonia, Epikeratoprosthesis

Leucocytes, v. Phagocytosis

Leukeran, v. Behcet's disease

Levator palpebrae, v. Lid loading

Leydhecker Test, v. Aqueous humour dynamics

Lichtkoagulation, v. Accidental coagulation, Argon laser, Automatic information, Diabetic

retinopathy, Photo-coagulation, Tunable laser

Lichtleiter-Laser, $v$. Tunable laser

Lid, v. Lid loading, Myxoma, Pterygium

Lid loading; upper lid loading with dermis graft and levator weakening 419

Linse, v. Artificial lens, Enucleation, Epikeratoprosthesis, Immunofluores-cence, Implant lens,

Lens research,

500

Index rerum ad Vol. 171

Mydriatiques, Optic approach, Peter's

anomaly, Phenylephrine, Secondary

glaucoma, Surgical anatomy,

Yellowing Lipid composition; the lipid composition

of aging sclera and cornea $82(\mathrm{~V})$ Lipidstoffwechsel, v. Fredrickson, Lipid

composition Livres nouveaux 176 (B), 244 (B) Lymphogranulomatose, v. Hodgkin's

disease 
Macula, v. Accidental coagulation, Diabetic retinopathy, Epitheliitis, Photocoagulation, Sparing of the macula, Stagnation thrombosis

Maculadegeneration, v. Retinal disturbances

Malformation, v. Anomalies

Medikament, v. Behcet's disease, Herpes keratitis, Herpetic keratitis, Hyper-parathyroidísm, Myambutol, Mydriatiques, Phenylephrine

Meerschweinchen, v. Phagocytosis

Meibom'sche Drüsen, v. Pterygium

Mesoptometry $39(\mathrm{~V})$

Metabolism, v. Fredrickson, Hyper-parathyroidism, Lipid composition, Zonular cataract

Metastatic carcinoma; electron microscopic demonstration of hormone-producing metastatic carcinoma of the choroid from the bronchus 451

Methode, v. Artificial lens, Depth acuity, Dog, Epikeratoprosthesis, Glaucoma-cataract surgery, Jones tube, Lid loading, Pump infusion, Rod response, Static perimetry, Visibility

Microscopie électronique,v. Metastatic carcinoma, Myxoma, Vitreous surgery

Mikrotrauma, v. Pterygium

Missbildung, v. Anomalies

Moraxella, v. Phagocytosis

Moraxella; exogenous Moraxella liquefaciens endophthalmitis 177

Mucocele $55(\mathrm{~V})$

Mucopolysaccharides, v. Vitreous surgery

Multiple stimulus, v. Static perimetry

Myambutol, v. Ethambutol

Myambutol; besteht Korrelation zwischen der Höhe der Myambutol-dosis und der Häufigkeit der Neuritis Nervioptici? 123

Mydriatiques; la prescription de mydriatiques dans la thérapie du glaucome par fermeture de Tangle 207

Myopia, v. Nyctalopia, Phenylephrine

Myxoma; a case of palpebral myxoma 488

Narkose, v. Halothannarkose

Nekrolog 405

Neovascularisation, v. Photocoagulation, Stagnation thrombosis

Nerf optique, v. Ethambutol, Myambutol

Netherlands Ophthalmological Society. 166th Meeting, Eindhoven 1971, 1-108(V)

Netherlands Ophthalmological Society. 167th Meeting, Vlissingen 1972 245-404(V)

Netzhaut, v. Accidental coagulation, Aqueous humour dynamics, Argon laser, Automatic

information, Behcet's disease, Cysticercus, Diabetic retinopathy, Dialysis, Epitheliitis, Glyco-

protein, Hodgkin's disease, Photocoagulation, Retinal disturbances, Rod response, Sponge

implants, Stagnation thrombosis, Trichomegaly, Tunable laser

Neuritis nervi optici, v. Behcet's disease, Ethambutol, Myambutol

Neurochirurgie, v. Fractures, Mucocele

Neuro-Ophthalmologie, v. Depth acuity, Sparing of the macula

Notfallsituationen am Auge 244 (B)

Nyctalopia; ophthalmologic and genetic study of a family with nyctalopia and myopia 358 (V)

Index rerum ad Vol. 171

501 
Nystagmography, v. Fixation

disturbances Nystagmus, v. Nyctalopia

Occlusion de Гartère centrale, v. Stagnation thrombosis

Oedem, v. Photocoagulation, Retinal disturbances

Open angle glaucoma, v. Aqueous humour dynamics, Pump infusion

Operation, v. Artificial lens, Cataract extraction, Cataract surgery, Corneal transplantation,

Cysticercus, Dog, Eaucleation, Epikeratoprosthesis, First extractions, Fractures, Fuchs' corneal

dystrophy, Glaucoma-cataract surgery, Jones tube, Lid loading, Mucocele, Secondary glaucoma, Sponge implants, Ultrasonography, Vitreous surgery

Ophthalmodynamometry with a suction-cup 50 (V)

Optic approach; physiologic optic approach of lens and cataract 249 (V)

Opticus, v. Ethambutol, Myambutol

Opticusatrophie, v. Myambutol

Optotype chart; a new optotype chart 380 (V)

Orbita, v. Fractures, Mucocele

Ordinateur, v. Automatic information, Campimeter, Implant lens

Outflow facility, v. Aqueous humour dynamics

Paedo-Ophthalmologie, v. Amblyopia, Halothannarkose, Zonular cataract

Parasit, v. Cysticercus, Hodgkin's disease

Paupière, v. Lid loading, Myxoma, Pterygium

Perimetrie, v. Accidental coagulation, Campimeter, Photocoagulation, Static perimetry, Visual acuity

Peter's anomaly $318(\mathrm{~V})$

Phagocytosis; problems in the in vitro methods of phagocytosis. Phagocytosis in pyridoxine deficiency $11(\mathrm{~V})$

Phenylephrine; severe myopia after pilocarpine in combination with phenylephrine eye drops 72

(V)

Photocoagulation, v. Accidental coagulation, Argon laser, Automatic information, Diabetic

retinopathy, Tunable laser

Photocoagulation in retinal vein thrombosis 365 (V)

Phthisis bulbi, v. Cyclocryoapplication

Pigment, v. Yellowing

Pigmentdegeneration, v. Aqueous humour dynamics, Trichomegaly

Pigmentepithel, v. Accidental coagulation, Argon laser, Epitheliitis, Hodgkin's disease,

Nyctalopia, Reticular structures, Retinal disturbances, Tunable laser

Pilocarpin, v. Phenylephrine, Pump infusion

Pinguekula, v. Pterygium

Pneumatonograph, v. Body position

Point de fuite, v. Argon laser

Poly I:C, v. Herpes keratitis, Herpetic keratitis

Position, v. Body position

Poule, v. Immunofluorescence

Proceedings, v. Netherlands Ophthalmological Society

Protanopie, v. Rod response

Protein, v. Glycoprotein, Immunofluorescence, Vitreous surgery 
Pseudophakie, v. Artificial lens, Determination, Dioptric power, Implant lens, Kataraktchirurgie, Ultrasonography

Pseudoxanthoma elasticum, v. Reticular structures

Pterygium; unsere weiteren Erfahrungen über die Pathogenese des Pterygiums 181

Pump infusion of pilocarpine 157

Pupillary bloc, v. Mydriatiques

Pyridoxin, v. Phagocytosis

Quellpunkt, v. Argon laser

502

Index rerum ad Vol. 171

Rabbit, v. Cyclocryoapplicatio,, Epikeratoprosthesis, Herpes keratitis, Tunable laser, Vitreous surgery

Radiogene Tumoren, v. Sarkom

Radiography; atlas of orbital radiography 244 (B)

Radiotherapie, v. Sarkom

Recherches expérimentales, v. Cyclo-cryoapplication, Herpes keratitis, Phagocytosis, Tunable

laser, Vitreous surgery

Refraction, v. Dioptric power, Implant lens, Optic approach

Reticular structures in the ocular fundus 67 (V)

Retina, v. Accidental coagulation, Aqueous humour dynamics, Argon laser, Automatic information, Behcet's disease, Cysticercus, Diabetic retino-pathy, Dialysis, Epitheliitis, Glycoprotein, Hodgkin's disease, Photo-coagulation, Retinal disturbances, Rod response, Sponge

implants, Stagnation thrombosis, Trichomegaly, Tunable laser

Retina; the vertebrate retina 176 (B)

Retinal detachment, v. Sponge implants

Retinal disturbances; significance of the configurations in retinal disturbances 321 (V)

Retinitis circinata, v. Retinal disturbances

Retinoblastom, v. Sarkom

Retinoblastoma; a study into the

possibility of differentiating hereditary from non-hereditary retinoblastoma $312(\mathrm{~V})$

Rétinopathie diabétique, v. Automatic information, Diabetic retinopathy, Glycoprotein

Retrobulbärneuritis, v. Ethambutol, Myambutol

Rod response; a simple method of differentiating the cone and rod responses in the ERG $328(\mathrm{~V})$

Ròntgenstrahlen, v. Sarkom

Sarkom; radiogenes Sarkom 12 Jahre nach Röntgenbestrahlung eines doppelseitigen

Retinoblastoms 109

Schielen, v. Nyctalopia

Schwerhörigkeit, v. Anomalies

Sclera, v. Lipid composition

Secondary glaucoma after lens extraction 255 (V)

Sehschärfe, v. Amblyopia, Optotype chart, Photocoagulation, Visual acuity

Sensibilité spectrale, v. Rod response, Standard observer

Serotonin, v. Metastatic carcinoma

Sinus frontalis, v. Mucocele

Sparing of the macula 91 (V) 
Spectral sensitivity, v. Rod response, Standard observer

Sponge implants; extraocular observations in episcleral sponge implants 439

Squint, v. Nyctalopia

Stäbchen, v. Rod response

Stagnation thrombosis. An iris fluorescein angiography study 192

Standard observer; the standard observer: a controversial subject 43 (V)

Static perimetry $26(\mathrm{~V})$

Stereopsis, v. Aniseikonia, Depth acuity, Kataraktchirurgie

Stimulation multiple, v. Static perimetry

Strabismus, v. Nyctalopia

'Student-assistancy'; increase of knowledge in 'student-assistants' during the 'studentassistancy' in ophthalmology at Leiden 382 (V)

Surdité, v. Anomalies

Surgery, v. Artificial lens, Cataract extraction, Cataract surgery, Corneal transplantation, Cysticercus, Dog, Enucleation, Epikeratoprosthesis, First extractions, Fractures, Fuchs' corneal dystrophy, Glaucoma-cataract surgery, Jones tube, Lid loading, Mucocele, Secondary glaucoma, Sponge implants, Ultrasonography, Vitreous surgery

Index rerum ad Vol. 171

503

Surgical anatomy of the lens and its

adnexa and some pathophysiological

remark* based on it 273 (V) Syndrome, v. Aqueous humour

dynamics, Trichomegaly Synéchie irido-lenticulaire,

v. Mydriatiques

Taenia solium, v. Cysticercus

Tapetoretinale Degeneration, v. Aqueous humour dynamics, Trichomegaly

Teaching, v. 'Student-assistancy'

Television du fond de $\Gamma æ i l, v$. Fixation disturbances, Fundus television

Tension oculaire, v. Aqueous humour dynamics, Body position, Cyclocryo-application,

Glaucoma-cataract surgery, Halothannarkose, Phenyl-ephrine, Pump infusion

Test, v. Amblyopia, Aqueous humour dynamics, Optotype chart, 'Student-assistancy'

Therapie, v. Argon laser, Behcet's disease, Diabetic retinopathy, Ethambutol, Herpes keratitis,

Herpetic keratitis, Hyperparathyroidism, Moraxella, Myambutol, Mydriatiques,

Photocoagulation, Pump infusion

Thrombose de la veine centrale, v. Photocoagulation, Stagnation thrombosis

Tiefenschärfe, v. Depth acuity

Tiger; clinical aspects and pathology of a Bengal tiger's eye 45 (V)

Tonography, v. Aqueous humour dynamics

Tonometrie, v. Aqueous humour dynamics, Body position, Halothannarkose, Pump infusion

Toxoplasmosis, v. Hodgkin's disease

Tränenwege, v. Jones tube

Transplantation, v. Lid loading

Trichomegaly; the syndrome of

trichomegaly, tapetoretinal degeneration and growth disturbances 313 (V)

Trigeminal zoster, v. Hodgkin's disease 
Troubles de la croissance,

v. Trichomegaly Tuberculosis, v. Ethambutol, Hodgkin's

disease, Myambutol Tumor, v. Metastatic carcinoma,

Myxoma, Retinoblastoma, Sarkom Tunable lasers in ophthalmology.

A waveguide dye laser photo-

coagulator 146

Ultrasonographie, v. Determination,

Implant lens Ultrasonography and lens implantation

276 (V) Unfall, v. Accidental coagulation Uveitis, v. Behcet's disease

Vaisseaux, v. Behcet's disease, Diabetic retinopathy, Fredrickson, Photocoagulation, Retinal

disturbances, Stagnation thrombosis

Varia 493

Vasculitis, v. Behcet's disease

VER, v. Accidental coagulation

Vererbung, v. Anomalies, Aqueous humour dynamics, Fredrickson, Fuchs' corneal dystrophy,

Nyctalopia, Reticular structure, Retinoblastoma, Trichomegaly

Vergilben, v. Yellowing

Verkalkung der Hornhaut, v. Hyperparathyroidism

Vessels, v. Behcet's disease, Diabetic retinopathy, Fredrickson, Photocoagulation, Retinal

disturbances, Stagnation thrombosis

Vieillissement, v. Lipid composition, Yellowing

Virus, v. Epitheliitis

Visibility, conspicuousness and attention 41 (V)

Vision des couleurs, v. Ethambutol

Vision en profondeur, v. Depth acuity

Visual acuity, v. Amblyopia, Optotype chart, Photocoagulation

Visual acuity visual field and physical ability. A proposal of a formula 100 (V)

504

Index rerum ad Vol. 171

Visual field, v. Accidental coagulation, Campimeter, Photocoagulation, Static perimetry, Visual acuity

Visually evoked response, v. Accidental coagulation

Vitreous body, v. Accidental coagulation, Cysticercus, Kataraktchirurgie, Vitreous surgery

Vitreous surgery; Donders lecture 1972. Biological factors in vitreous surgery 390 (V)

Voies lacrymales, v. Jones tube

Vorderkammer, v. Artificial lens, Determination, Dioptric power, Enucleation, Glaucoma-

cataract surgery, Mydriatiques, Stagnation thrombosis, Ultrasonography

Wachstumsstörungen, v. Trichomegaly Waveguide dye laser, v. Tunable laser

Weitwinkelglaukom, v. Aqueous humour

dynamics, Pump infusion Winkelblockglaukom, v. Mydriatiques

Yellowing; factors in the yellowing process of the human lens 247 (V)

Zapfen, v. Rod response

Zentralarterienverschluss, v. Stagnation thrombosis

Zentralvenenthrombose, v. Photo-coagulation, Stagnation thrombosis

Ziliarkörper, v. Cyclocryoapplication 
Zonular cataract develops as a

complication of a disturbance in the carbohydrate metabolism in the neonatal or infantile phase of life $254(\mathrm{~V})$

Z. Zoster, v. Hodgkin's disease

Zytostatica, v. Behcet's disease 